\title{
LA PLANIFICACIÓN ESTRATÉGICA E INDICADORES DE CALIDAD EDUCATIVA
}

\author{
GUSTAVO HERNÁNDEZ CASTRO \\ Universidad Estatal a Distancia, Costa Rica \\ ghernandezc@uned.ac.cr \\ JENNY FERNÁNDEZ JINESTA \\ Colegio Saint Francis, Costa Rica \\ jfinesta@hotmail.com
}

\section{RESUMEN}

Este artículo presenta los resultados obtenidos a partir de la aplicación de una matriz de indicadores de calidad educativa, desde la planificación estratégica. La matriz en cuestión se utilizó en trece instituciones de educación en el año 2016. Es una investigación exploratoria, en el cual el método de trabajo de campo ha sido seleccionado como el más eficaz para conocer a mayor profundidad la aplicabilidad del modelo del diagnóstico de calidad.

Se analiza el modelo de calidad mediante las percepciones y aspiraciones de los sujetos que participan en la investigación y mediante la recolección de registrosy documentos relevantes para lograr comprender desde una perspectiva holística la problemática o necesidad educativa institucional en la aplicabilidad del modelo.

PALABRAS CLAVE: CALIDAD EDUCATIVA, SOSTENIBILIDAD, ADMINISTRACIÓN, ESTRATEGIAS, MODELO EDUCATIVO.

\begin{abstract}
This article presents the results obtained from the application of a matrix of indicators of educational quality, from strategic management. The matrix was applied to thirteen institutions of education in the year 2016. It is an exploratory investigation, in which the Field research has been selected as the most effective to know in greater depth the applicability of the quality diagnosis model. The quality model is analyzed through the perceptions and aspirations of the subjects involved in the research and through the collection of relevant records and documents, to understand from a holistic perspective the institutional educational problem or need in the applicability of the model.
\end{abstract}

KEYWORDS: EDUCATIONAL QUALITY, SUSTAINABILITY, MANAGEMENT, STRATEGIES. 


\section{INTRODUCCIÓN}

El tema central de este artículo es la aplicabilidad de una matriz de indicadores de calidad educativa que facilitan la evaluación de las áreas organizativa-administrativas, de sostenibilidad y validación de procesos en las instituciones educativas. La gestión educativa, visualizada en una red compleja de subprocesos, debe iniciar desde la planeación estratégica, ya que esta permite construir en las entidades educativas una cultura de planificación para la consecución de los objetivos educacionales de la nación y de las comunidades anfitrionas que nutren a los centros educativos de su insumo más preciado, que es el estudiantado (Llorente, 2010). En consecuencia, las instituciones educativas cultivan a las generaciones que son las que construyen y desarrollan al país, elevando de esta forma, las capacidades sociales del Estado (Sen, 2000).

Establecido este vínculo, se define la planificación estratégica como el proceso mediante el cual los diferentes niveles de la institución educativa se ajustan para que las acciones emprendidas con- tribuyan al logro de las metas de la organización. La planificación estratégica como producto de un ejercicio de reflexión transdisciplinario debe incorporar la calidad para que las instituciones educativas construyan una cultura de mejora continua (Álvarez, Topete, y Abundes, 2011).

En este contexto, la planificación estratégica está vinculada al proceso administrativo: planear, organizar, dirigir y controlar. Este proceso se logra utilizando con efectividad recursos no humanos (insumos, aulas, bibliotecas, instalaciones deportivas, etc.) y trabajando con personas (estudiantado, cuerpo docente y administrativo), motivándolas para hacer realidad las metas de la organización (Castillo, 2005).

En consecuencia, la planificación estratégica (Fuster, 2008), incorpora los criterios de la filosofía organizacional (visión-misión, objetivos, políticas y valores institucionales, entre otras, propios de cada cultura organizacional). La gestión administrativa y académica, la sostenibilidad, la calidad y, como ejes transversales, el estudiantado, el profesorado, la familia y el país, ver figura 1.

FIGURA 1

\section{PLANEACIÓN ESTRATÉGICA Y CALIDAD EN LA EDUCACIÓN}

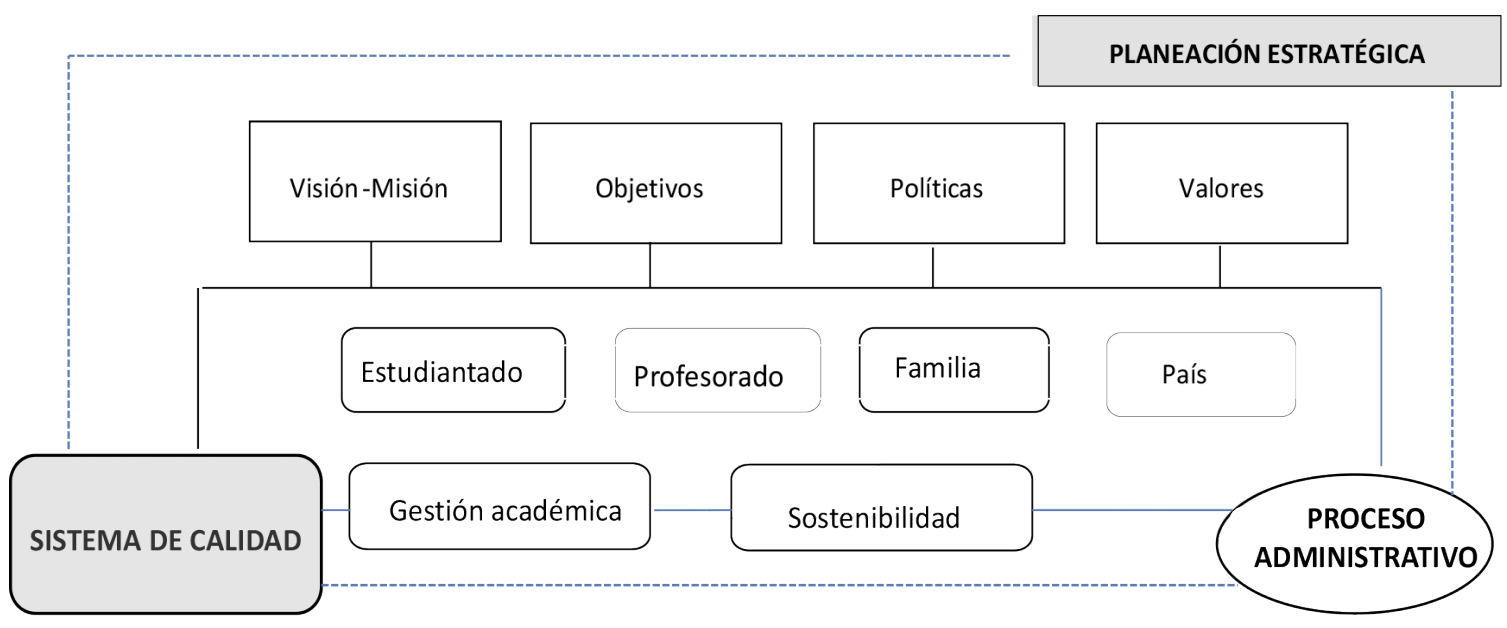

Fuente: Hernández y Fernández (2016). 
Como se indicó, la planificación estratégica está vinculada a la administración educativa; la estrecha relación entre ambas permite la construcción de una subjetividad que modela una persona capaz de contribuir a elevar las capacidades sociales y espirituales de la nación. Lo anterior se logra mediante la integración de esfuerzos organizacionales, que desde la filosofía institucional son el sustento ontológico de las organizaciones (ver figura 2).

En esa dirección, las instituciones educativas deben contar con un sistema e indicadores de calidad para gestionar asertivamente sus procesos de sostenibilidad administrativa y académica de forma eficaz. Esto con la finalidad de que sean herramientas para la toma de decisiones, a partir de la planificación estratégica (OEl, 2010).

La matriz de indicadores de calidad, validadas en la investigación, es el resultado de la reflexión de una praxis sobre la calidad en la educación realizada en trece instituciones educativas, con el objetivo de proporcionar una herramienta de fácil aplicación al gerente educativo, para que mediante un liderazgo proactivo diagnostique la gestión administrativa y académica. La matriz incorpora los criterios, indicadores y predictores más relevantes de la planificación estratégica, la administración educativa, la sostenibilidad y la calidad académica.

Los sistemas de evaluación de sistemas educativos cada vez son más exigentes, ya que el entorno exige a los futuros profesionales contar con las competencias que requieren para desempeñarse adecuadamente en el mundo profesional (Tiana, 2014). Por otra parte, estos sistemas de evaluación requieren indicadores de calidad puntuales, para determinar si los sistemas de evaluación, corresponden o no, las exigencias del mercado laboral (Fonseca, Oviedo, Hernández, Pérez, y Zawalinski, 2014).

FIGURA 1

PLANEACIÓN ESTRATÉGICA Y ADMINISTRACIÓN EDUCATIVA

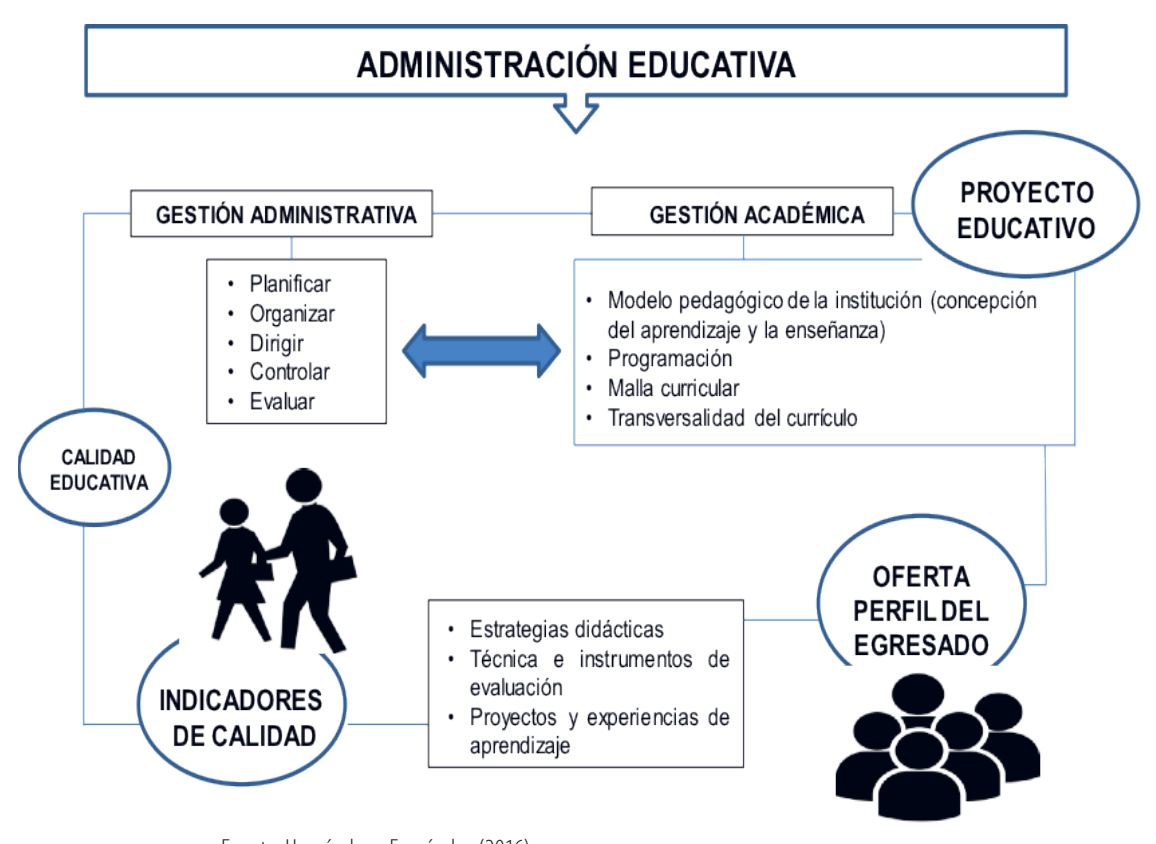

Fuente: Hernándezy Fernández (2016). 


\section{METODOLOGÍA}

Es una investigación exploratoria, el método de trabajo de campo ha sido seleccionado como el más eficaz para conocer a mayor profundidad la aplicabilidad de la matriz de indicadores de calidad (Soto-Lesmes y Durán de Villalobos, 2010). A través de la observación del entorno y la participación en él, en este trabajo, se analiza el problema detectado mediante las percepciones y aspiraciones de los sujetos que participan en la investigación y mediante la recolección de registros y documentos relevantes para lograr comprender desde una perspectiva holística la problemática o necesidad educativa institucional (Corral, 2009).

Este accionar es compatible con el diseño de investigación abordado porque favorece el descubrimiento de respuestas a fenómenos sociales respecto a lo que ocurre en un centro educativo (Serbia, 2007). Su explicación procede de la interpretación de los datos y no a partir de supuestos, para así obtener un diagnóstico real de las instituciones analizadas (Cuñat, 2007).

En el caso particular de esta investigación, comprende analizar los resultados de la aplicación de la Matriz de indicadores de la calidad educativa, permitiendo visualizar sus fenómenos y problemáticas, que conllevan a los sujetos de investigación a ser los involucrados directos en el día a día de las instituciones y que son los que llevan a la práctica la planificación estrategia en todos los niveles organizativos (Sánchez, 2011). En otras palabras, se "estudia a los objetos y seres vivos en sus contextos o ambientes naturales y cotidianidad" (Hernández, Fernández, y Baptista, 2010, p. 10). La investigación exploratoria trata de comprender la situación o fenómeno tal y como se presenta, libre de prejuicios para que las interpretaciones emanen de los eventos objetivamente (Gurdián-Fernández, 2007).

El enfoque investigativo de este trabajo es fundamentalmente cualitativo (Sánchez, 2005). La im- portancia de la adopción del enfoque cualitativo se apoya en la capacidad que este fundamento metodológico ofrece para recolectar datos sobre la complejidad de las organizaciones educativas.

La Matriz de indicadores de la calidad educativa posee tres dimensiones (Organización, Sostenibilidad y Validación de procesos); a su vez, cada dimensión la conforman diez variables que son el sustento para realizar el diagnóstico de administrativo, académico y de calidad. Por su parte, cada variable tiene veintidós indicadores y estos suman setenta y seis predictores, que especifican lo que se desea medir. La tabla 1 permite visualizar la estructura resumida de la matriz.

Se diseñó un instrumento basado en la escala de Likert (Schwarzer y Jerusalem, 1995) para otorgarle un valor cualitativo a cada predictor $y$, de esta forma, establecer el grado de satisfacción obtenido. La escala de valor está distribuida de uno a cinco, donde 1 y 2 se consideran valores negativos; 3 representa un valor intermedio; 4 y 5 establecen un nivel de medición satisfactorio. La tabla 2, describe la escala aplicada en el diagnóstico.

Para esta investigación, se ha seleccionado un grupo representativo de trece instituciones educativas con el fin de conocer la aplicabilidad de la Matriz de indicadores de la calidad educativa. Por tal motivo, es un muestreo no probabilístico; esto quiere decir que cada unidad de análisis es intencionalmente seleccionada por sus posibilidades de ofrecer información profunda y detallada sobre los fenómenos de investigación (Martínez-Salgado, 2012). El objetivo primordial no es medir cuantitativamente, sino lograr entender los acontecimientos y procesos de calidad en toda su complejidad que se presentan en los centros educativos.

El levantamiento de los datos se recolectó entre los meses de febrero de 2014 a noviembre de 2016 (realización de diez talleres) en trece instituciones educativas del país conformadas de la siguiente manera: 
TABLA 1

DIMENSIÓN, VARIABLES, INDICADORES, PREDICTORES

\begin{tabular}{|c|c|c|c|}
\hline DIMENSIÓN & VARIABLES & INDICADORES & PREDICTORES \\
\hline \multirow[t]{3}{*}{ Organización } & Gestión administrativa & 4 & 17 \\
\hline & Gestión académica & 4 & 17 \\
\hline & Gestión humana & 4 & 18 \\
\hline \multirow[t]{3}{*}{ Sostenibilidad } & Desempeño ambiental & 3 & 5 \\
\hline & Infraestructura & 2 & 2 \\
\hline & Vinculación comunitaria & 1 & 13 \\
\hline \multirow[t]{5}{*}{ Validación de procesos } & Programas de auditoría & 1 & 1 \\
\hline & Acciones correctivas & 1 & 1 \\
\hline & Indicadores de desempeño & 1 & 1 \\
\hline & Indicadores de servicio & 1 & 1 \\
\hline & 10 & 22 & 76 \\
\hline
\end{tabular}

Fuente: Hernández y Fernández (2016)

TABLA 2

\section{CRITERIO Y ESCALA DE EVALUACIÓN}

\begin{tabular}{|l|l|}
\hline Criterio & Escala \\
\hline Nunca & 1 \\
\hline Parcialmente & 2 \\
\hline Ocasionalmente & 3 \\
\hline Frecuentemente & 4 \\
\hline Muy frecuentemente (somos un ejemplo a seguir en el sector educativo) & 5 \\
\hline
\end{tabular}

Fuente: Hernández y Fernández (2016)

- Dos instituciones educativas públicas: Educación I y || ciclos (| || C)

- Una institución educativa privada: Educación I y || ciclos (| || CP)

- Dos colegios técnicos públicos: Especialidades Técnicas (CT)

- Cuatro colegios públicos: Educación III ciclo y Educación Diversificada (III C ED)

- Dos universidades públicas (UP)

- Una Escuela Municipal de Música (EM)
- Un Departamento de Educación Técnica (gubernamental) (ETG)

La Matriz de indicadores de la calidad educativa tiene la estructura que se observa en la Tabla 3.

Como se indicó, la matriz la conforman tres dimensiones (Organización, Sostenibilidad y Validación de procesos). A su vez, cada dimensión tiene sus variables propias e indicadores que permiten diagnosticar cada área de la institución educativa, donde se debe desarrollar las actividades para implementarla.

Cada indicador tiene una serie de predictores que diagnostican y permiten implementar las 
TABLA 3

MATRIZ DE INDICADORES DE CALIDAD EDUCATIVA [RESUMIDA]

\begin{tabular}{|c|c|c|c|}
\hline \multicolumn{2}{|r|}{ Gestión administrativa } & \multirow{15}{*}{ Dimensión organizacional } & \multirow{29}{*}{$\begin{array}{l}\text { MATRIZ DE INDICADORES } \\
\text { CALIDAD EDUCATIVA }\end{array}$} \\
\hline 1.1 & Planificación estratégica & & \\
\hline 1.2 & Estructura organizacional & & \\
\hline 1.3 & Proceso administrativo & & \\
\hline 1.4 & Comunicación interna & & \\
\hline \multicolumn{2}{|r|}{ Gestión académica } & & \\
\hline 1.5 & Gestión docente & & \\
\hline 1.6 & Evaluación de los aprendizajes (EA) & & \\
\hline 1.7 & Validación de EA & & \\
\hline 1.8 & Promoción académica & & \\
\hline \multicolumn{2}{|r|}{ Gestión humana } & & \\
\hline 1.9 & Competencias docentes & & \\
\hline 1.10 & Actualización docente & & \\
\hline 1.11 & Clima organizacional & & \\
\hline 1.12 & Salud y bienestar & & \\
\hline \multicolumn{2}{|r|}{ Desempeño ambiental } & \multirow{9}{*}{ Dimensión sostenibilidad } & \\
\hline 1.13 & Impacto ambiental en la comunidad & & \\
\hline 1.14 & Manejo de desechos & & \\
\hline 1.15 & Programa de consumo (energía, otras) & & \\
\hline \multicolumn{2}{|r|}{ Infraestructura } & & \\
\hline 1.16 & Mantenimiento preventivo & & \\
\hline 1.17 & Mantenimiento correctivo & & \\
\hline \multicolumn{2}{|r|}{ Vinculación comunitaria } & & \\
\hline 1.18 & Programas de extensión & & \\
\hline \multicolumn{2}{|r|}{ MEJORA } & \multirow{5}{*}{$\begin{array}{l}\text { Dimensión validación de } \\
\text { procesos }\end{array}$} & \\
\hline 1.19 & Programas de auditoría & & \\
\hline 1.20 & Acciones correctivas & & \\
\hline 1.21 & Indicadores de desempeño & & \\
\hline 1.22 & Indicadores de servicio & & \\
\hline
\end{tabular}

Fuente: Hernández y Fernández (2016) 
mejoras que se necesiten. Por ejemplo, la dimensión organización consta de tres variables (administración, gestión académica y gestión humana) y cada variable tiene sus indicadores y predictores. En el caso de la dimensión Organización, la variable administración tiene cuatro indicadores, a saber: planificación estratégica, estructura organizacional, proceso administrativo y comunicación interna. Estos tienen predictores que, en el caso del indicador denominado planificación estratégica, consta de 7 predictores (tabla 3 [resumida]).

Las variables son propiedades, identificadores o características de la realidad que permiten conocer el estado existente de una situación en particular. Por ejemplo, en el modelo de calidad (tabla 3 [resumida] y tabla 7 [full]), la variable administración permitirá conocer si la institución educativa tiene o no una estructura organizacional necesaria para llevar su planificación y gestión académica y, en consecuencia, la implementación de las acciones administrativas y académicas.

Los predictores, son acciones que ayudan a prevenir situaciones antes de que ocurran; por ejemplo, la definición de la estructura organizacional (organigrama), la institución debe tener como mínimo la ubicación de todos los puestos en un organigrama. Como se puede observar, se aprecia cómo, por cada variable, se instituyen los indicadores que la institución educativa debe respaldar a través de procedimientos escritos, los cuales son la columna vertebral del sistema de calidad. De tal forma, si la institución no tiene por escrito y puestos en evidencia los predictores establecidos en la matriz, mediante manuales, procedimientos, protocolos u otro tipo de documentos, significa que el predictor establecido no se realiza. En otras palabras, en cuestión de calidad: lo que no está escrito, no se hace.

Durante la etapa de la recolección de la información se combinaron técnicas e instrumentos del enfoque cualitativo como lo son: Delphi, talleres, entrevistas en profundidad y observación participativa, lo cual permitió sistematizar el proceso y la aplicación de la Matriz de indicadores de calidad educativa. El diseño y aplicación de esta matriz, consistió en trabajar en cuatro etapas.

La primera etapa fue elegir, mediante un muestreo no probabilístico, trece instituciones de educación. Una vez seleccionados los centros educativos, se realizó un taller con los respectivos directores generales y coordinadores académicos, para presentarles el propósito de la investigación, la metodología de trabajo, la explicación de la Matriz de indicadores de calidad educativa y la calendarización para realizar quince talleres de trabajo.

La segunda etapa consistió en ejecutar los doce talleres (8 horas de duración cada uno, según cronograma acordado). Se les solicitó a los participantes (directores y coordinadores académicos de los centros educativos escogidos) trabajar bajo la actividad brainstorming para la depuración de la Matriz indicadores de calidad educativa conformada por un total de 650 predictores. Con la aplicación de esta técnica, cada institución participante escogió 50 predictores de calidad que abarcaran las tres dimensiones. Posteriormente $y$, en tres talleres adicionales, se procedió a conjuntar y mejorar los predictores según cada dimensión, cada variable y cada indicador. Del total de 650 predictores, se obtuvo un listado final de 76 predictores, que permitieron elaborar en forma definitiva la Matriz de indicadores de calidad educativa (tabla 7 [full]).

La tercera etapa se fundamentó en la aplicación de la técnica Delphi a un panel de expertos (personas ajenas a las instituciones educativas escogidas) en los temas de Sistemas de calidad educativa y Sistema de aseguramiento de calidad. El objetivo fue obtener un conceso de criterio especialista respecto al tema investigado (Landeta, 2002), es decir, sobre Matriz de indicadores de calidad educativa. De las ventajas de 
la aplicación de la técnica Delphi se destacan el anonimato, la retroalimentación controlada por los investigadores y la respuesta estadística de grupo (Cortés e Iglesias, 2005).

Los investigadores elaboraron una lista de 16 candidatos y candidatas expertos que cumplieran con los siguientes criterios de argumentación (requisitos):

- Análisis teórico realizado por el experto (publicaciones en revistas científicas)

- Experiencia en el área de conocimiento (consultorías)

- Investigaciones de autores nacionales que conoce

- Investigaciones de autores internacionales que conoce

- Conocimientos propios acerca del tema

- Know how (experiencia en calidad educativa)

A continuación, se determinó el coeficiente de competencia según la siguiente fórmula:

\section{$\mathrm{K}$ comp. $=1 / 2(\mathrm{Kc}+\mathrm{Ka})$}

Coeficiente de competencia de cada experto
Donde:

Kc: Coeficiente de conocimiento

Ka: Coeficiente de argumentación

Para establecer el $\mathrm{K}$ comp. $=1 / 2(\mathrm{Kc}+\mathrm{Ka})$, se diseñó y aplicó una encuesta de estructurada con 20 preguntas (según los requisitos establecidos) para que, de forma individual, cada experto respondiera y otorgara a cada una de las preguntas, un valor, según el conocimiento personal. El coeficiente es el resultado del promedio de los valores que cada experto concedió en la respuesta a las preguntas.

Para determinar el Ka, se suman de los valores del grado de influencia de cada uno de los criterios de argumentación con respecto a una tabla diseñada para ordenar los datos obtenidos de la encuesta (ver tabla 4). El experto anotó el grado de influencia de cada uno de los criterios, según la escala: alta competencia, media competencia o baja competencia. Se suman los valores obtenidos y el resultado derivado representa el Ka obtenido por cada experto. Dados los coeficientes Kc y Ka, se calculan para cada experto y se obtiene el valor de K comp:

\begin{tabular}{|l|l|}
\hline La competencia del experto es ALTA & si K comp. $>0.8$ \\
\hline La competencia del experto es MEDIA & si $0.5<K$ comp. $>=0.8$ \\
\hline La competencia del experto es BAJA & si $K$ comp. $<=0.5$ \\
\hline
\end{tabular}

TABLA 4

INFLUENCIA SEGÚN CRITERIOS DE ARGUMENTACIÓN

\begin{tabular}{|l|c|c|c|}
\hline \multicolumn{1}{|c|}{ Criterio de argumentación (requisitos) } & Alta competencia & Media competencia & Baja competencia \\
\hline Análisis teórico realizado por el experto & 0.3 & 0.2 & 0.1 \\
\hline Experiencia en el área de conocimiento (consultorías) & 0.5 & 0.4 & 0.2 \\
\hline Investigaciones de autores nacionales que conoce & 0.05 & 0.04 & 0.03 \\
\hline Investigaciones de autores internacionales que conoce & 0.05 & 0.04 & 0.03 \\
\hline Conocimientos propios acerca del tema & 0.05 & 0.04 & 0.03 \\
\hline Know how (experiencia en calidad educativa) & 0.05 & 0.04 & 0.03 \\
\hline
\end{tabular}

Fuente: Hernández y Fernández, a partir de los datos de Cortés e Iglesias 2005. 
De esta forma, y de un total de 16 especialistas, se eligieron siete expertos que obtuvieron la calificación de alta competencia. Es importante señalar que los expertos seleccionados no se conocieron entre sí. La tabla 5 muestra el perfil de los expertos seleccionados.

La tabla 6 muestra los resultados obtenidos de los siete expertos seleccionados según el alto nivel de competencia (Kcomp>0.8), lo cual permitió que cada especialista examinara la Matriz de indicadores de calidad educativa, y procediera a emitir un criterio técnico mediante una secuencia de validación establecida. Esto con la finalidad de enriquecer la matriz y con esto perfeccionarla para su posterior aplicación en los centros educativos seleccionados.
La cuarta etapa consistió en que los lideres educativos de las instituciones educativas seleccionadas aplicaran la matriz diseñada en sus respectivas instituciones, con el propósito de validarla y obtener una percepción del grado de madurez acerca de la organización, la administración y de la calidad del centro educativo. Es menester aclarar que, a solicitud de los distintos representantes legales de las instituciones educativas en las que se les aplicó la matriz, se omite el nombre jurídico de estas y solamente se menciona la actividad desempeñada que realiza cada centro educativo. Asimismo, los investigadores dejan abierta la posibilidad a los interesados en la temática, de solicitar información de la base de datos disponible, que sustentan los datos de la matriz aplicada y que alimentaron el desarrollo y las posteriores conclusiones que arrojó la pesquisa.

TABLA 5

\section{PERFIL DE EXPERTOS}

\begin{tabular}{|l|l|}
\hline Experta 1 & Auditora certificada en Normas de Aseguramiento de Calidad ISO 9000 \\
\hline Experta 2 & Directora de Colegio Público con 25 años de experiencia en dirección \\
\hline Experto 3 & Administrador Educativo con 25 años de experiencia en Educación privada \\
\hline Experto 4 & Par acreditado en Sistema Nacional de Acreditación de la Educación Superior (SINAES) \\
\hline Experta 5 & Profesora universitaria en cursos de calidad en la educación y Currículo \\
\hline Experta 6 & Profesora en enseñanza I y II público con 15 años de experiencia \\
\hline Experto 7 & Director de Colegio en Educación Técnica 10 años de experiencia \\
\hline
\end{tabular}

TABLA 6 CÁLCULO DEL COEFICIENTE DE COMPETENCIA DE CADA EXPERTO

\begin{tabular}{|c|c|l|c|c|}
\hline Experto & Kc & \multicolumn{1}{|c|}{ Ka } & K comp & Calificación \\
\hline 1 & 1 & $0.5+0.3+4(0.05)=1$ & 1 & Alta \\
\hline 2 & 1 & $0.5+0.2+4(0.05)=0.9$ & 0.95 & Alta \\
\hline 3 & 0.9 & $0.2+0.5+0,04+3(0,05)=0.89$ & 0.895 & Alta \\
\hline 4 & 0.8 & $0,5+0,2+0.05+0.04+2(0.03)=0.85$ & 0.83 & Alta \\
\hline 5 & 0.9 & $0.2+0.5+4(0.04)=0.76$ & 0.86 & Alta \\
\hline 6 & 0.8 & $0.3+0.5+0.03+0.04+0.05+0.04=0.96$ & 8.88 & Alta \\
\hline 7 & 0.9 & $0.3+0.5+2(0.04)+0.05+0.04=0.97$ & 0.935 & Alta \\
\hline
\end{tabular}




\section{DESARROLLO}

A continuación, se presentan los datos obtenidos del proceso de aplicación de la Matriz de indicadores de calidad educativa según las concordancias encontradas en las trece instituciones participantes. Se omiten los resultados particulares de cada institución, la información recopilada tiene un alto impacto a lo interno de cada centro educativo, y por la confidencialidad del mismo, no puede transcribirse para la presentación de este artículo. No obstante, y para los distintos gestores educativos, la Matriz de indicadores de calidad educativa contribuyó a que los participantes diseñaran planes estratégicos institucionales con el propósito de subsanar las debilidades y posibles mejoras que la misma matriz suministró.

\section{Dimensión Organización}

Los predictores de mayor coincidencia en las 13 instituciones donde se aplicó el modelo en la dimensión de Organización, fueron los siguientes: O2, O8, 011, 014, 015, 022, O25, O33 Y O42 (ver figura 3).
En la figura 3, se observa cómo los predictores O2, O8 y 042 tienen en común, que para una sola institución educativa (privada), se realizan las gestiones de forma parcial, ocasional y frecuente, según cada caso. Los restantes predictores para el restante de los centros educativos examinados, muestran que las gestiones nunca se realizan. Analizando con más detalle estos resultados se tiene que:

1. Se deben mejorar los mecanismos de la información para la solución de problemas y consultas del estudiantado.

2. Se debe mejorar el enlace entre la administración y docentes, padres de familia y estudiantes. No todos los sectores se encuentran satisfechos con la gestión administrativa.

3. La toma de decisiones es unilateral, no existe la participación del personal.

4. Se debe fortalecer la gestión administrativa, delimitar, aclarar y comunicar mejor al equipo toda la información; además, trabajar en la planeación estratégica e involucrar más al personal en la toma de decisiones y formulación de metas.

FIGURA 3

\section{DIMENSIÓN ORGANIZACIÓN}

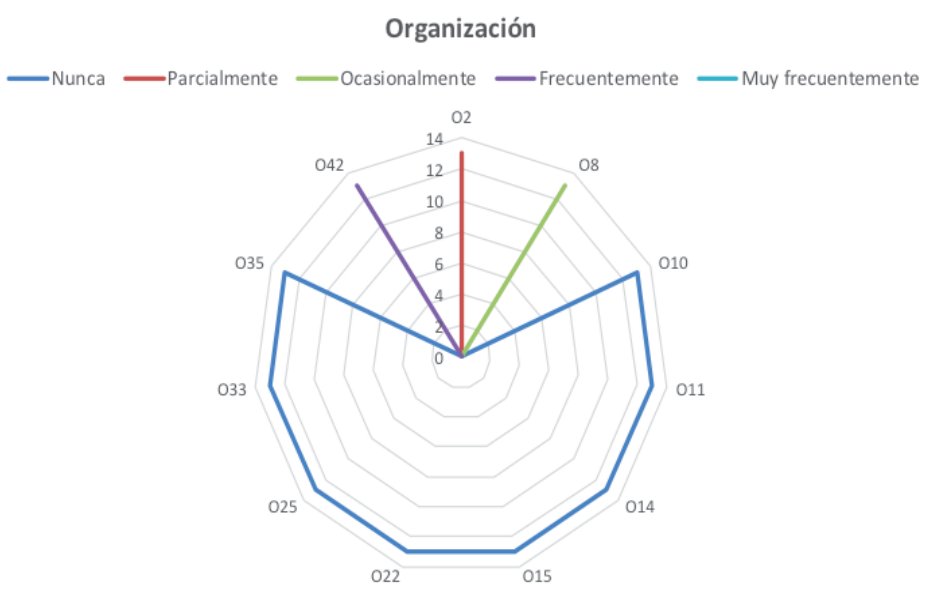

Fuente: Hernández y Fernández (2016). 
5. Motivar más al equipo para aumentar sus conocimientos, participar de actividades de actualidad educativa y buscar nuevas estrategias de enseñanza, creativas e innovadoras.

6. Se resaltan las diferencias entre el centro educativo y el resto de las entidades que conforman el sistema educativo. Esta realidad hace pensar que, eventualmente, el Ministerio de Educación Pública (MEP) deberá hacer ciertos ajustes para aplicar herramientas evaluativas en las instituciones educativas.

7. La satisfacción del personal docente es débil. Los recargos administrativos inciden en un clima laborar negativo. Las personas que tienen estos puestos a cargo, manifiestan un exceso de trabajo y que no existe una claridad en la delimitación de sus funciones, ya que cumplen responsabilidades docentes y al mismo tiempo, administrativas.

8. La institución es subvencionada por el Estado, lo que ocasiona una maraña legal; ya que existen funcionarios nombrados y pagados por el MEP y tutelados por la Ley de Carrera Docente y otros que son funcionados pagados por la institución, que se rigen por el Código de Trabajo. Pero en las funciones de recargos administrativos, existen personas que son del MEP y al mismo tiempo, asumen responsabilidades con recargo de parte de la administración de la institución. Esto, provoca disconformidad ante la duda de cuál es la relación laboral que se establece en ambas situaciones.

\section{Dimensión Sostenibilidad}

Respecto a la dimensión de Sostenibilidad, los predictores de mayor coincidencia en las 13 instituciones analizadas se presentan en dos extremos. Por un lado, en la institución educativa (privada) se realizan gestiones frecuentemente según los predictores S59, S68 y S70. En los predictores S54, S57, S58, S59, S68 y S70 de las restantes 12 instituciones educativas nunca no se realizan las gestiones pertinentes según el modelo aplicado (ver figura 4).

\section{FIGURA 4 \\ DIMENSIÓN SOSTENIBILIDAD}

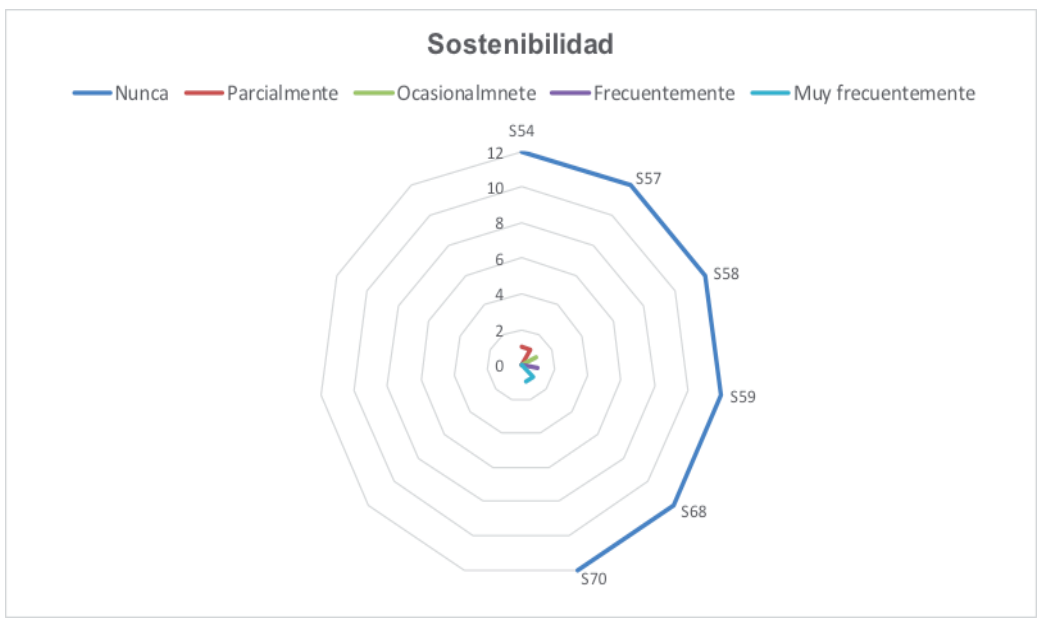

Fuente: Hernández y Fernández (2016). 
Analizando con más detalle estos resultados, en relación con la Dimensión de Sostenibilidad, la información sustantiva señala que:

1. En aspectos de proyección a la comunidad no se realizan muchas intervenciones, son parte de las carencias de las acciones que desarrolla la institución.

2. Si bien existe un programa de reciclaje, en la escuela no se maneja un programa para el manejo de los desechos.

3. Se utilizan los servicios de la municipalidad.

4. Los padres de familia no se involucran con los programas de sostenibilidad.

5. No se refleja en la institución un sólido programa de sostenibilidad. Existe el programa de bandera azul, que funciona en muchos lugares y se realiza con éxito. El programa no abarca aspectos más relevantes que se presentan en los indicadores del modelo.

6. Se implementan mecanismos para el ahorro del agua, proyectos de reciclaje con los estudiantes y buena disposición del comité institucional de bandera azul.

7. Es necesario confeccionar protocolos que unifiquen y sistematicen los procesos que se dan en la institución. El ideal es que todos los funcionarios tengan el conocimiento y sobre los procedimientos a ejecutar.

8. No hay ningún protocolo para medir, evaluar o controlar el desempeño de las educadoras en general. Es una debilidad, se está haciendo un esfuerzo para mejorar.

9. De los veinte predictores analizados, siete obtuvieron la respuesta Prácticamente no se realiza. Estos responden a las variables "Desempeño ambiental" e "Infraestructura", lo cual llama la atención sobre todo si se considera que la institución está inmersa en una comunidad en la cual el gobierno local ha sido incapaz de manejar el asunto de la recolección de basura y la inseguridad ciudadana.

\section{Dimensión Validación de procesos}

Al analizar los datos obtenidos en la Dimensión Validación de procesos, los predictores de mayor coincidencia en las 13 instituciones analizadas son similares que en los resultados emanados en la dimensión anterior.

Por un lado, en la institución educativa (privada), se realizan frecuentemente las gestiones según el predictor V73, V75 y V76, y los predictores las restantes 12 instituciones educativas en la cuales prácticamente nunca se ejecutan las gestiones pertinentes según la Matriz de indicadores de calidad educativa aplicada (figura 5).

Al examinar en detalle los resultados obtenidos se aprecia que:

1. En el análisis y valoración de los resultados, se han dejado de realizar programas de proyección hacia la comunidad producto de la inseguridad ciudadana. Se deben diseñar distintas estrategias para fortalecer la relación con la comunidad.

2. La institución cuenta con la normativa interna que establece los deberes y derechos de la comunidad educativa. No obstante, se evidencia una carencia en la aplicación de la normativa y pasan de ser simplemente directrices; no se establecen con claridad los procedimientos a seguir.

3. En la dimensión Validación de procesos, la respuesta para los cuatro predictores, referentes a auditoría, acciones correctivas, desempeño y servicio, fue la más baja "Prácticamente no se realiza". Se debe iniciar con la implementación del control interno que contribuya con el ordenamiento de la in- 


\section{FIGURA 5 \\ DIMENSIÓN VALIDADCIÓN DE PROCESOS}

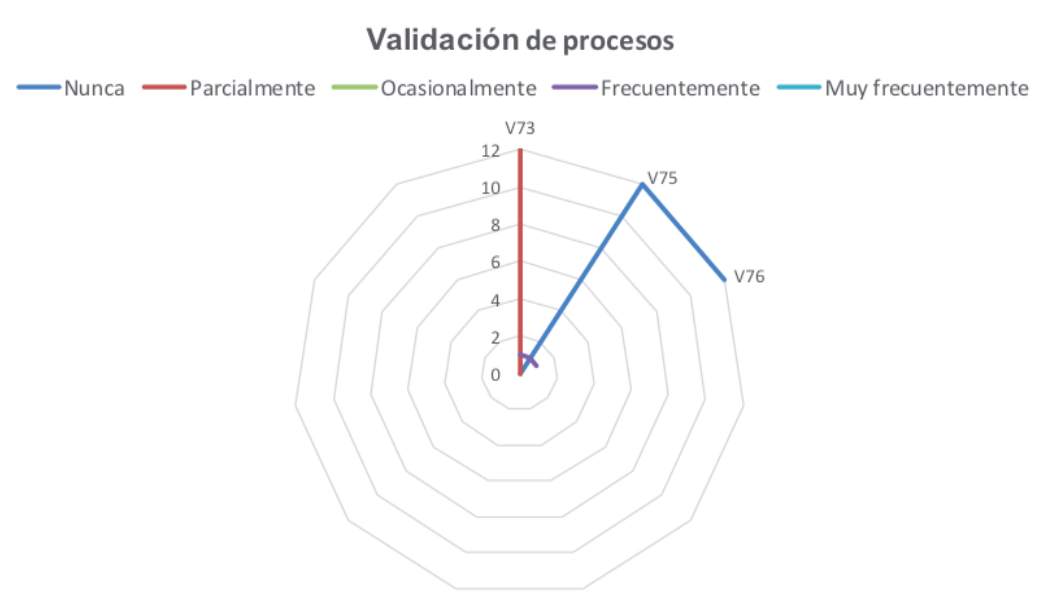

Fuente: Hernández y Fernández (2016).

formación y los documentos propios de la gestión administrativa y académica.

\section{CONCLUSIONES}

De la aplicación de la Matriz de indicadores de calidad educativa, se desprende que es un instrumento eficaz para medir el grado de madurez organizacional, administrativa, académica y de calidad de una institución educativa. A su vez, con los insumos obtenidos de la aplicación de la matriz, se facilita el diseño del plan estratégico de la institución, en el cual es indispensable alinear la filosofía organizacional o institucional (visión-misión, objetivos, políticas, valores) con los ejes transversales (estudiantado, profesorado, familia y país) vinculados con la gestión académica y la sostenibilidad. De esta, forma la organización, es decir, las instituciones educativas, pueden responder integralmente a las necesidades educativas y económicas de la nación.

Al contrastar el grado de respuestas en cada dimensión según su variable, indicador y predictor, le permite al gerente educativo iniciar un proceso de planificación estratégica (figura 1) con el objetivo de empoderar a las organizaciones de una alta competencia administrativa y académica. En los resultados obtenidos se aprecia que los gestores administrativos (gerentes educativos) no lideran sus organizaciones de una forma eficiente y participativa y, en consecuencia, la insatisfacción del personal docente y administrativo incide en el desempeño institucional.

Una de las fortalezas de la Matriz de indicadores de calidad educativa es su aplicabilidad para cualquier tipo de organización educativa, es decir, su flexibilidad permite recolectar información de instituciones educativas privadas, públicas o mixtas, tanto de centros de educación de enseñanza primaria, secundaria y universitaria. El modelo reúne la información indispensable para que el gestor educativo formule un plan estratégico y operativo que le permita cumplir con la normativa de las respectivas leyes nacionales, pero fundamentalmente, con iniciar procesos de fortalecimiento de la gestión académica. 
Al compartirlo con los colaboradores de la organización, el modelo posibilita al gestor educativo conocer la percepción de sus colaboradores respecto a la gestión que se realiza (retroalimentación) y respecto a la vinculación con la comunidad anfitriona. Estas acciones le facilitaran al gerente educativo diseñar estrategias particulares de intervención para el mejoramiento de la calidad institucional en sus tres dimensiones.

El establecimiento de manuales, procedimientos, expedientes, estadísticas, etc., es fundamental para constituir instituciones educativas con grado de madurez óptimo para la consecución de sus objetivos administrativos y académicos y, alienados estos, con la planificación estratégica institucional. Todos los participantes de la investigación coinciden en la necesidad de aplicar Matriz de indicadores de calidad educativa dos veces al año; al inicio y cierre del curso lectivo. Lo anterior para crear y fortalecer en la institución una cultura de calidad permanente.

\section{REFERENCIAS}

Álvarez, I., Topete, C., y Abundes, A. (2011). El concepto emergente de gestión estratégica y desafíos para la formación en gestión (pp. 1-11). México: Escuela Superior de Comercio y Administración, Instituto Politécnico Nacional. Recuperado de http:// www.comie.org.mx/congreso/memoriaelectronica/v11/docs/area_13/1466.pdf

Castillo, A. (2005). Liderazgo administrativo: Reto para el director de escuelas del siglo XXI. Cuaderno de Investigación en la Educación, (20), 34-51.

Cortés, M. E., y Iglesias, M. (2005). Generalidades sobre Metodología de la Investigación. México: UNACAR.

Corral, Y. (2009). Validez y confiabilidad de los instrumentos de investigación para la recolección de datos. Ciencias de la educación, 19(33), 229-247.

Cuñat, R. (2007). Aplicación de la teoría fundamentada (Grounded Theory) al estudio del proceso de creación de empresas. En Decisiones basadas en el conocimiento y en el papel social de la empresa:
XX Congreso anual de AEDEM (pp. 44-50). Madrid: Asociación Europea de Dirección y Economía de Empresa.

Fonseca, C., Oviedo, C., Hernández, F., Pérez, G., y Zawalinski, L. (2014). Modelo de Evaluación de la Calidad de la Educación Costarricense (MECEC) (3era ed.). San José: MEP Dirección de Gestión y Evaluación de la Calidad.

Fuster, J. (2008). La planificación estratégica: Una propuesta metodológica para gestionar el cambio en políticas de innovación educativa. Revista lberoamericana de Educación, 46(1), 1-11.

Gurdián-Fernández, A. (2007). El Paradigma Cualitativo en la Investigación Socio-Educativa. San José: PrintCenter.

Hernández, R., Fernández, C., y Baptista, L. (2010). Metodología de la investigación. México: McGrawHill.

Landeta, J. (2010). El método Dep/hi. Buenos Aires: Ariel.

Lorente, Á. (2010). Tema 5: Estructura y cultura organizativa de los centros educativos. En Experto Universitario en Administración de la Educación. CADE 20082009 (pp. 1-27). Madrid: MEC-OEI-UNED.

Martínez-Salgado, C. (2012). El muestreo en investigación cualitativa: principios básicos y algunas controversias. Ciência y Saúde Coletiva, 17(3), 613-619.

Organización de Estados Iberoamericanos para la Educación, la Ciencia y la Cultura (OEI). (2010). Significado y alcance de las metas educativas: ¿ $\mathrm{Ha}$ cia dónde queremos ir juntos? En Metas Educativas 2021 (pp. 85-143). Madrid: CUDIPAL.

Rodríguez de Torrealba, Á. (2008). Hacia una visión prospectiva de la gerencia y la supervisión educativa. Revista Investigación y Posgrado, 23(2), 447-461.

Sánchez, M. (2011). La metodología en la investigación cualitativa. Mundo siglo XXI, (1), 115-118.

Schwarzer; R., y Jerusalem, M. (1995). Generalized Self-Efficacy scale. In J. Weinman, S. Wright, \& M. Johnston (Eds), Measures in health psicologic: A user portafolio. Casual and control beliefs (pp. 3537). Windosr. UK: NEFER-NELSON 
Sen, A. (2002). Rationality and Freedom. Cambridge: Harvard University Press.

Serbia, J. (2007). Diseño, muestreo y análisis en la investigación cualitativa. Hologramática, 23(3), 123-146.

Soto-Lesmes, V., y Durán de Villalobos, M. (2010). El trabajo de campo: Clave de la investigación cualitativa. Aquichan, 10(3), 253-266.
Triana, A. (2014). Evaluación y cambio educativo: Los debates actuales sobre las ventajas y los riesgos de la evaluación. En E. Martín y F. Martínez (Eds.), Avances y desafíos en la evaluación educativa (pp. 17-28). Madrid: Fundación Santillana.

Recibido: 22 de setiembre de 2017

Aceptdo:10 de diciembre de 2017 
TABLA 7

MATRIZ DE INDICADORES DE CALIDAD EDUCATIVA [VERSIÓN FULL]

\begin{tabular}{|c|c|c|c|c|c|c|c|}
\hline & & sca & & & Dimensión & Predictor & Códino \\
\hline 1 & 2 & 3 & 4 & 5 & organizacional & & couigo \\
\hline & & & & & & Funciones, puestos y estructura organizacional debidamente definidos & 01 \\
\hline & & & & & & Conocimiento del personal de la estructura organizacional establecida & 02 \\
\hline & & & & & (estructura organizacional) & Satisfacción del personal de la estructura organizacional establecida & 03 \\
\hline & & & & & & Conocimiento del personal de la planeación estratégica institucional & 04 \\
\hline & & & & & & Satisfacción del personal con la visión-misión institucional & 05 \\
\hline & & & & & & Satisfacción del profesorado con los valores de la institución & 06 \\
\hline & & & & & Gestión administrativa & Participación del personal para la toma de decisiones en la institución & 07 \\
\hline & & & & & (planeación estratégica) & Revisión anual de la planeación estratégica (o parte de esta) & 08 \\
\hline & & & & & & Satisfacción del profesorado con las políticas y objetivos institucionales & 09 \\
\hline & & & & & & $\begin{array}{l}\text { Satisfacción de la Ley } 7372 \text { con la ejecución de los recursos económicos gestiona- } \\
\text { dos por la administración }\end{array}$ & 010 \\
\hline & & & & & & Medición del tiempo para dar respuesta a las expectativas del estudiantado & 011 \\
\hline & & & & & Gestión administrativa & Satisfacción del personal docente con recargos administrativos & 012 \\
\hline & & & & & & Satisfacción de los estudiantes con el servicio de comedor & 013 \\
\hline & & & & & (proceso duminIIIstiativo) & Índices actualizados de ausentismo, deserción estudiantil & 014 \\
\hline & & & & & & Mantener información suficiente y necesaria & 015 \\
\hline & & & & & & Garantizar que se atendieron todas las consultas y dudas el estudiantado & 016 \\
\hline & & & & & (comunicación interna) & Satisfacción del estudiantado por el servicio recibido & 017 \\
\hline & & & & & & Satisfacer las demandas ofrecidas en los programas curriculares & 018 \\
\hline & & & & & & Satisfacción de los contenidos por los docentes & 019 \\
\hline & & & & & & Satisfacción de los contenidos por el estudiantado & 020 \\
\hline & & & & & & Solventar adecuadamente las inquietudes y preguntas del estudiantado & 021 \\
\hline & & & & & (gestión docente) & Satisfacción del estudiantado con la metodología de evaluación & 022 \\
\hline & & & & & & Satisfacción de los estudiantes con el servicio ofrecido por la coordinación del área & 023 \\
\hline & & & & & & Motivación de los estudiantes que llevan materias atrasadas o que adelantan & 024 \\
\hline & & & & & & $\begin{array}{l}\text { Satisfacción del estudiantado con las herramientas de la evaluación de los } \\
\text { aprendizajes }\end{array}$ & 025 \\
\hline & & & & & (evaluación aprendizajes) & Satisfacción de los estudiantes que cursan el año completo en sus materias & 026 \\
\hline
\end{tabular}




\begin{tabular}{|c|c|c|c|c|c|c|c|}
\hline \multicolumn{5}{|c|}{ Escala } & \multirow{2}{*}{$\begin{array}{l}\text { Dimensión } \\
\text { organizacional }\end{array}$} & \multirow{2}{*}{ Predictor } & \multirow{2}{*}{ Código } \\
\hline 1 & 2 & 3 & 4 & 5 & & & \\
\hline & & & & & \multirow{4}{*}{$\begin{array}{l}\text { Gestión académica } \\
\text { (validación evaluación } \\
\text { aprendizajes) }\end{array}$} & $\begin{array}{l}\text { Satisfacción de los estudiantes con las herramientas tecnológicas implementadas } \\
\text { en las lecciones }\end{array}$ & 027 \\
\hline & & & & & & Satisfacción de los estudiantes con los ítems de evaluación aplicados & 028 \\
\hline & & & & & & $\begin{array}{l}\text { Porcentaje de estudiantes que recibieron el temario de peritazgo, con al menos } \\
\text { cinco meses previos a la aplicación de la prueba }\end{array}$ & 029 \\
\hline & & & & & & $\begin{array}{l}\text { Satisfacción del estudiantado con la formación técnica recibida por los docentes de } \\
\text { especialidad, previa a la práctica profesional }\end{array}$ & 030 \\
\hline & & & & & \multirow{4}{*}{$\begin{array}{l}\text { Gestión académica } \\
\text { (promoción académica) }\end{array}$} & 100\% de promoción estudiantil en todos los niveles & 031 \\
\hline & & & & & & Parámetros aceptables de ausentismo & 032 \\
\hline & & & & & & No tolerancia a la deserción estudiantil & 033 \\
\hline & & & & & & $\begin{array}{l}\text { Satisfacción del estudiantado con el apoyo del docente para la preparación de } \\
\text { repasos previos a las pruebas nacionales de peritazgo }\end{array}$ & 034 \\
\hline & & & & & \multirow{5}{*}{$\begin{array}{c}\text { Gestión humana } \\
\text { (competencias docentes) }\end{array}$} & Producción intelectual (al menos una publicación por año por cada docente) & 035 \\
\hline & & & & & & Grado académico mínimo de Licenciatura & 036 \\
\hline & & & & & & Participación anual en proyectos de vinculación con el contexto educativo & 037 \\
\hline & & & & & & Participación en comisiones temáticas de la institución & 038 \\
\hline & & & & & & Probadas competencias en dominio de idiomas (profesores de idiomas) & 039 \\
\hline & & & & & \multirow{3}{*}{$\begin{array}{l}\text { Gestión humana } \\
\text { (actualización docente) }\end{array}$} & Participación en actividades de capacitación en su área de conocimiento por año & 040 \\
\hline & & & & & & Participación en eventos académicos por año & 041 \\
\hline & & & & & & Participación en redes académicas & 042 \\
\hline & & & & & \multirow{5}{*}{$\begin{array}{c}\text { Gestión humana } \\
\text { (clima organizacional) }\end{array}$} & Mantener una actitud de respeto y amabilidad & 043 \\
\hline & & & & & & $\begin{array}{l}\text { Satisfacción del personal docente que viene a la institución menos de un día o que } \\
\text { son itinerantes }\end{array}$ & 044 \\
\hline & & & & & & Motivación del personal de limpieza para que las instalaciones estén siempre limpias & 045 \\
\hline & & & & & & Motivación del personal administrativo que se encuentra reubicado en la institución & 046 \\
\hline & & & & & & Actitud de respeto y amabilidad (no se aceptan maltratos de palabras y gestos) & 047 \\
\hline & & & & & \multirow{5}{*}{$\begin{array}{l}\text { Gestión humana } \\
\text { (salud y bienestar) }\end{array}$} & Instalaciones limpias y ordenadas & 048 \\
\hline & & & & & & Programas de comunicación constantes de prevención en salud & 049 \\
\hline & & & & & & Programas de capacitación constantes de prevención en salud & 050 \\
\hline & & & & & & Equipos de trabajo conformados para atender casos de accidentes & 051 \\
\hline & & & & & & $\begin{array}{l}\text { Apoyo a las situaciones especiales del personal (familiares, salud, económicas, } \\
\text { situacionales, etc.) }\end{array}$ & 052 \\
\hline
\end{tabular}




\begin{tabular}{|c|c|c|c|c|c|c|c|}
\hline & & scalc & & & DIMENSIÓN & Predictor & Códian \\
\hline 1 & 2 & 3 & 4 & 5 & SOSTENIBILIDAD & Prealctor & Codigo \\
\hline & & & & & & Programas establecidos de sostenibilidad en la institución & S53 \\
\hline & & & & & $\begin{array}{l}\text { ambiental (impacto ambien- } \\
\text { tal en comunidad) }\end{array}$ & Programas establecidos de sostenibilidad proyectados en las comunidades & S54 \\
\hline & & & & & Sostenibilidad. Desempeño & Programa establecido para el manejo desechos & S55 \\
\hline & & & & & $\begin{array}{l}\text { ambiental (manejo de } \\
\text { emisiones y desechos) }\end{array}$ & Programa establecido para el reciclaje & S56 \\
\hline & & & & & $\begin{array}{l}\text { Sostenibilidad. Desempeño } \\
\text { ambiental (consumo de agua, } \\
\text { luz, otros) }\end{array}$ & Programa establecido para el ahorro del consumo de agua, luz, otros & S57 \\
\hline & & & & & $\begin{array}{l}\text { Sostenibilidad. Infraestructura } \\
\text { (mantenimiento preventivo) }\end{array}$ & $\begin{array}{l}\text { Programa establecido de mantenimiento preventivo para la sostenibilidad } \\
\text { ambiental }\end{array}$ & S58 \\
\hline & & & & & $\begin{array}{l}\text { Sostenibilidad. Infraestructura } \\
\text { (mantenimiento correctivo) }\end{array}$ & Programa establecido de mantenimiento correctivo de los activos de la institución & S59 \\
\hline & & & & & & Satisfacción de los vecinos por los programas realizados por la institución en la comunidad & S60 \\
\hline & & & & & & $\begin{array}{l}\text { Estudiantes contratados por los empresarios durante el periodo de práctica } \\
\text { profesional }\end{array}$ & S61 \\
\hline & & & & & & Cantidad de estudiantes enviados a pasantías en empresas afines con la especialidad & S62 \\
\hline & & & & & & $\begin{array}{l}\text { Satisfacción de los estudiantes con las actividades extracurriculares programadas } \\
\text { en la institución }\end{array}$ & $\$ 63$ \\
\hline & & & & & & Satisfacción de la dirección con los comités institucionales & 564 \\
\hline & & & & & Sostenibilidad. Vinculación & Sensibilidad del educador para con las necesidades socioculturales del estudiante & S65 \\
\hline & & & & & comunitaria (programas de & Satisfacción de los padres (encargado) con los valores que inculca la institución & S66 \\
\hline & & & & & extemsion y ue empleos & $\begin{array}{l}\text { Satisfacción de los padres (encargado) con la inversión que implica la permanencia } \\
\text { del estudiantado en la institución }\end{array}$ & 567 \\
\hline & & & & & & Satisfacción de los padres (encargado) con el desempeño del director (a) & S68 \\
\hline & & & & & & Satisfacción de los padres (encargado) con el desempeño del personal administrativo & $\$ 69$ \\
\hline & & & & & & Satisfacción de los padres (encargado) con el desempeño del personal docente & S70 \\
\hline & & & & & & Satisfacción de los padres (encargado) con el desempeño del estudiantado & S71 \\
\hline & & & & & & Satisfacción de los padres (encargado) con la evaluación de la calidad institucional & $\$ 72$ \\
\hline 1 & 2 & scalc & 4 & 5 & $\begin{array}{l}\text { DIMENSIÓN } \\
\text { VALIDACIÓN } \\
\text { DE PROCESOS }\end{array}$ & Predictor & Código \\
\hline & & & & & Programas de auditoría & Procedimientos escritos en todas las áreas de la organización & S73 \\
\hline & & & & & $\begin{array}{l}\text { Programa de acciones } \\
\text { correctivas }\end{array}$ & $\begin{array}{l}\text { Procedimientos de acciones correctivas para todas las áreas de la organización: } \\
\text { fechas, acciones, costos, responsables }\end{array}$ & S74 \\
\hline & & & & & Protocolos de desempeño & Protocolos de medición del desempeño óptimo de la organización & S75 \\
\hline & & & & & Protocolos de servicio & $\begin{array}{l}\text { Protocolos de medición de indicadores de calidad educativos: por actividad, } \\
\text { estructura organizacional y responsabilidad }\end{array}$ & S76 \\
\hline
\end{tabular}

